\title{
Research
}

Carolina Guiriguet, Laura Muñoz-Ortiz, Andrea Burón, Irene Rivero, Jaume Grau, Carmen Vela-Vallespín, Mercedes Vilarrubí, Miquel Torres, Cristina Hernández, Leonardo Méndez-Boo, Pere Toràn, Llorenç Caballeria, Francesc Macià and Antoni Castells

\section{Alerts in electronic medical records to promote a colorectal cancer screening programme:}

\author{
a cluster randomised controlled trial in primary care
}

\begin{abstract}
Background

Participation rates in colorectal cancer screening are below recommended European targets.

\section{Aim}

To evaluate the effectiveness of an alert in primary care electronic medical records (EMRs) to increase individuals participation in an organised, population-based colorectal cancer screening programme when compared with usual care.
\end{abstract}

\section{Design and setting}

Cluster randomised controlled trial in primary care centres of Barcelona, Spain.

\section{Method}

Participants were males and females aged 50-69 years, who were invited to the first round of a screening programme based on the faecal immunochemical test (FIT) ( $n=41$ 042), and their primary care professional. The randomisation unit was the physician cluster $(n=130)$ and patients were blinded to the study group. The control group followed usual care as per the colorectal cancer screening programme. In the intervention group, as well as usual care, an aler to health professionals (cluster level) to promote screening was introduced in the individual's primary care EMR for 1 year. The main outcome was colorectal cancer screening participation at individual participant level.

\section{Results}

In total, 67 physicians and 21619 patients

(intervention groupl and 63 physicians and 19423 patients (control group) were randomised. In the intention-to-treat analysis screening participation was $44.1 \%$ and $42.2 \%$ respectively lodds ratio $1.08,95 \%$ confidence interval $[\mathrm{Cl}]=0.97$ to 1.20 , $P=0.146$ ). However, in the per-protocol analysis screening uptake in the intervention group showed a statistically significant increase, after adjusting for potential confounders (OR, 1.11 $95 \% \mathrm{Cl}=1.02$ to $1.22 ; P=0.018$ )

\section{Conclusion}

The use of an alert in an individual's primary care EMR is associated with a statistically significant increased uptake of an organised, FIT-based colorectal cancer screening programme in patients attending primary care centres.

\section{Keywords}

colorectal neoplasm; early detection of cancer: electronic medical record; mass screening; primary health care; reminder systems.

\section{INTRODUCTION}

Colorectal cancer has the highest incident rate of all cancers and is the second leading cause of cancer death in Western countries. ${ }^{1}$ Evidence from several studies have demonstrated that colorectal cancer screening is effective ${ }^{2-5}$ and cost effective ${ }^{6}$ in populations that are at average risk of developing the disease. Searching for occult blood in stools using the guaiac test and, more recently, the faecal immunochemical test (FIT) are predominantly implemented in Europe ${ }^{7}$ and Australia ${ }^{8}$, whereas colonoscopy is the dominant screening modality in North America?. Moreover, at population-based level, implementation of organised programmes, in contrast to opportunistic screening, is recommended because they include an administrative structure responsible for service delivery, quality assurance and evaluation.?

The European guidelines for quality assurance in colorectal cancer screening states that the participation rate represents a key quality indicator for ensuring effectiveness and efficiency of populationbased screening programmes. ${ }^{7}$ However, results from current programmes in Spain

C Guiriguet, MD, primary care physician; I Rivero MD, primary care physician; C Vela-Vallespín, MD primary care physician; $\mathbf{M}$ Vilarrubí, MD, primary care physician, Catalan Health Institute, Santa Coloma de Gramenet, Spain. L Méndez-Boo, MPH, preventive medicine and public health specialist, University Institute in Primary Care Research Jord Gol, Barcelona, Spain. L Muñoz-Ortiz, statistician; $\mathbf{P}$ Toràn, MD, primary care physician and head of research support unit, Metropolitana Nord: L Caballeria, $\mathrm{PhD}, \mathrm{MD}$, primary care physician, University Institute in Primary Care Research Jord Gol, Santa Coloma de Gramenet, Spain. A Burón, $\mathrm{PhD}, \mathrm{MPH}, \mathrm{MD}$, preventive medicine and public health specialist; $\mathbf{C}$ Hernández, data manager; F Macià, MD, preventive medicine and public health specialist, Hospital del Mar Medical Research Institute, Barcelona, Spain. J Grau, MD, preventive medicine and public health specialist, preventive Medicine and Hospital Epidemiology Department, Hospital Clinic, University of Barcelona, Spain show that participation is not reaching these recommended figures, with uptake rates varying from $17.2 \%$ to $42.3 \%,{ }^{10-14}$ with the exception of the Basque Country (64.3\%). ${ }^{14}$ The uptake rate in the first round for the Colorectal Cancer Screening Program of Barcelona (CCSPB) was 43.6\%. ${ }^{15}$ Differences in programme organisation, sociodemographics, and primary care professional involvement may explain these differences (for example, the involvement of the GP differs depends on certified training. scheduling of colonoscopy, information feedback, or FIT collection in primary care).

The CCSPB is targeted at males and females aged 50-69 years, who are at average risk of developing colorectal cancer; it encompasses a total population of 197795 individuals. ${ }^{15}$ Individuals receive a mailed invitation letter, along with an explanatory leaflet about colorectal cancer, screening, and the specific programme. The FIT is delivered and collected at the community pharmacies involved in the programme.

Participants with a positive test (cut-off level of $20 \mu \mathrm{g}$ of haemoglobin/g of faeces) are invited, by a trained nurse from the

M Torres, PhD, MD, internal medicine specialist, Department of Internal Medicine, Fundació Hospital de L'Esperit Sant, University of Barcelona, Spain. A Castells, PhD, MD, gastroenterology specialist and medical director, Hospital Clinic, University of Barcelona, Spain

\section{Address for correspondence}

Carolina Guiriguet, Catalan Health Institute, Santa Rosa Primary Care Center, Carrer El Cano s/n, Santa Coloma de Gramenet, 08923 Spain.

E-mail: cguiriguet.bnm.icsdagencat.cat

Submitted: 3 December 2015; Editor's response: 25 December 2015; final acceptance: 25 February 2016.

\section{CBritish Journal of General Practice}

This is the full-length article (published online 7 Jun 2016) of an abridged version published in print. Cite this version as: $\mathbf{B r} \mathbf{J}$ Gen Pract 2016; DOI: 10.3399/bjgp16X685657 


\section{How this fits in}

Participation rates in colorectal cancer screening are below recommended targets in many European countries. Incorporating alerts into electronic medical records (EMRs) has proved effective for improving the practice of different preventive care measures, including cancer screening, although limited information has been reported about colorectal cancer, especially in the context of populationbased screening programmes. This study presents a clinical trial performed within an organised colorectal cancer screening programme with participation of community pharmacies for the delivering of faecal immunochemical tests. A significant increase in colorectal cancer screening uptake was found among patients attending primary care centres. GPs play a key role in promoting colorectal cancer screening, facilitated by their proximity and relationship with patients, but in busy practices it could be difficult to recommend or order cancer screening. Efforts should continue to develop interventions involving GPs within the population-based programmes.

screening office, to undergo colonoscopy. Both primary care professionals and pharmacists attended a specific training session at the beginning of each screening round, which explained their role and the functioning of the programme, for example, the standard procedures outlined in the CCSPC. ${ }^{15}$ Primary care professionals are encouraged to promote colorectal cancer screening and to help the programme refine the target population by identifying individuals with exclusion criteria.

Direct recommendation by the GP has been identified as one of the strongest predictors for colorectal cancer screening uptake. ${ }^{16}$ Data obtained in Catalonia indicated that $88.9 \%$ of individuals would agree to participate in a colorectal cancer screening programme if their primary care professional suggested it; ${ }^{17}$ this figure, however, is far from the actual participation rates. In Catalonia, public primary care is organised through primary care centres that each cover a specific population area. Every individual is assigned to a primary care unit, where one physician and one nurse attend to their own patients. Although controversy exists about prompting health professionals to promote colorectal cancer screening, ${ }^{18}$ the use of new technological strategies may help to increase participation in such programmes. ${ }^{19}$ In fact, the introduction of specific reminders in electronic medical records (EMRs) has proved effective for improving a range of preventive care measures, including cancer screening. ${ }^{20}$ However, despite its potential high impact and low cost, electronic reminders remain underutilised in healthcare.

The aim of this study was to evaluate the effectiveness of an electronic alert in individuals' primary care EMR to increase their participation in an organised, FITbased colorectal cancer screening programme.

\section{METHOD}

The Colo-Alert study was a single-blinded, cluster randomised controlled trial comparing an electronic alert to primary care professionals in individual's EMR to increase uptake in an organised, FIT-based colorectal cancer screening programme to usual care of a primary care professional. It was performed within the population-based CCSPB.

This project was registered at ClinicalTrials.gov (NCT01877018), and the study protocol published elsewhere. ${ }^{21}$

\section{Sample and recruitment}

The study was performed in 10 primary care centres. Participants were males and females aged $50-69$ years, who were invited to the first round of the CCSPB and had a GP assigned to their participating centre, together with their respective primary care professional. Primary care centres were successively involved in the CCSPB; the recruitment period ran from July 2011 until May 2012 . Follow-up lasted for 1 year after invitation for each centre. Figure 1 outlines the study process.

Patient characteristics were obtained from the EMR and provided by the Primary Care Services Information System at the beginning of the study. Patients baseline characteristics are summarised in Table 1. Anonymity of data was guaranteed throughout the whole study process.

The control group comprised all study participants assigned to primary care professionals who did not receive any electronic reminder to increase participation; they, therefore, followed the standard CCSPB protocol..$^{15}$

\section{Interventions and procedures}

All study participants assigned to health professionals who received an alert to increase participation constituted the intervention group. In addition to the standard CCSPB procedures, an electronic reminder was included in individuals' primary care EMR for 1 year, identifying those subjects who had been invited to the 


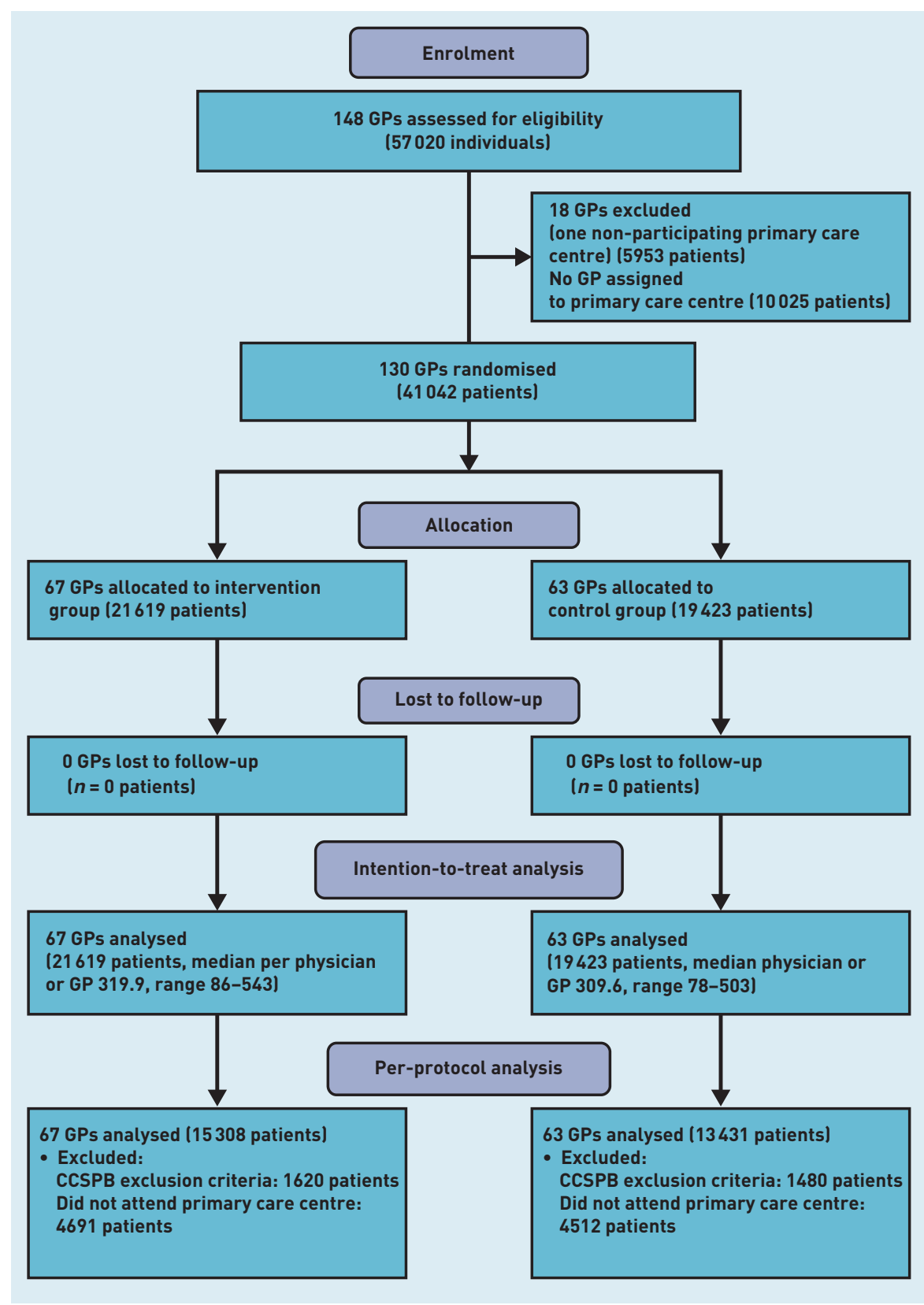

Figure 1. CONSORT flowchart of the study process. CCSPB $=$ Colorectal Cancer Screening Program of Barcelona
CCSPB lintervention targeted at cluster levell.

When a non-participant of the CCSPB was identified in any medical appointment, both GPs and nurses were asked to encourage participation by means of brief, structured counselling.

The socioeconomic deprivation index was expressed as quintiles: the first quintile (Q1) represents the least deprived while the fifth quintile $(Q 5)$ represents the most deprived. ${ }^{22}$ The clinical risk group, defined in nine initial categories, was collapsed into two categories:

- without dominant chronic illness (healthy acute illness, minor chronic illness, or multiple minor chronic illness); and

- with dominant chronic illness lone dominant chronic illness, two chronic illnesses, three chronic illnesses, neoplasm, or catastrophic illness). ${ }^{23}$

\section{Outcomes}

The outcome of the study was participation in the CCSPB, which was defined as individuals returning the FIT during the follow-up period. At the beginning of the study, the CCSPB technical office provided a list of individuals invited to participate in the programme and their corresponding primary care centre; at the end of study, it provided information regarding participation.

\section{Sample size}

The appropriateness of including the entire population (130 GPs with 41042 patients, belonging to primary care centres whose patients were invited to participate in the CCSPB from July 2011 until May 2012) was considered. This sample size allowed the smallest increase in participation - 3.7 percentage points between the two groups - to be detected, taking into account the clusters and assuming a low colorectal cancer screening uptake rate of $30 \%$ in the control group.

An intracluster correlation coefficient (ICC) of $\rho=0.022$ was assumed with an average cluster size of 318.2. This resulted in a design effect of eight; dividing the intended sample size (41 042 patients) by the design effect resulted in an effective sample size of 2565 patients in each group. An $\alpha$ risk of 0.05 and $\beta$ risk of 0.20 was assumed.

\section{Randomisation}

The GP (cluster) was the unit of randomisation. Allocation of GP to the intervention or control group was carried out through a random allocation (1:1 ratio). Nurses were allocated to the intervention and control group according to the allocation of the corresponding GP with whom they shared a patient's care. If a nurse shared a patient's care with a GP allocated to a different study group, the nurse was allocated to the group of the GP with which they shared most patients. Patients were allocated to the intervention or control group according to the allocation of their corresponding GP.

Given the nature of the intervention, it was not possible to carry out blinding of the primary care professionals but patients 
Table 1. Participants' baseline characteristics

\begin{tabular}{|c|c|c|c|}
\hline Characteristics & $\begin{array}{c}\text { Total } \\
n=41042\end{array}$ & $\begin{array}{c}\text { Intervention } \\
n=21619\end{array}$ & $\begin{array}{c}\text { Control } \\
n=19423\end{array}$ \\
\hline \multicolumn{4}{|l|}{ Sex, $n(\%)$} \\
\hline Male & 19039 (46.4) & $9971(46.1)$ & 9068 (46.7) \\
\hline Female & $22003(53.6)$ & 11648 (53.9) & 10355 (53.3) \\
\hline Mean age, years (SD) & $58.7(5.6)$ & $58.8(5.6)$ & $58.7(5.6)$ \\
\hline \multicolumn{4}{|l|}{ Age groups, $n(\%)$} \\
\hline $50-54$ & 11991 (29.2) & 6195 (28.7) & 5796 (29.8) \\
\hline $55-69$ & 10765 (26.2) & 5590 (25.9) & $5175(26.6)$ \\
\hline $60-64$ & $9910(24.1)$ & 5411 (25.0) & 4499 (23.2) \\
\hline $65-69$ & $8376(20.4)$ & 4423 (20.5) & $3953(20.4)$ \\
\hline \multicolumn{4}{|l|}{ Smoking, $n[\%]$} \\
\hline Never smokers & 14028 (34.2) & 7429 (34.4) & 6599 (34.0) \\
\hline Smokers & 6908 (16.8) & 3703 (17.1) & 3205 (16.5) \\
\hline Ever smokers & 8105 (19.7) & 4507 (20.8) & 3598 (18.5) \\
\hline Unknown & 12001 (29.2) & $5980(27.7)$ & $6021(31.0)$ \\
\hline \multicolumn{4}{|l|}{ Alcohol consumption, $n(\%)$} \\
\hline Non drinkers & 16352 (39.8) & 8792 (40.7) & 7560 (38.9) \\
\hline Low-risk drinkers & 9349 (22.8) & 5008 (23.2) & 4341 (22.3) \\
\hline High-risk drinkers & 760 (1.9) & $413(1.9)$ & $347(1.8)$ \\
\hline Unknown & 14581 (35.5) & 7406 (34.3) & 7175 (36.9) \\
\hline \multicolumn{4}{|l|}{ Body mass index, $n(\%)$} \\
\hline Low weight & 539 (1.3) & 284 (1.3) & 255 (1.3) \\
\hline Normal weight & 5351 (13.0) & 2847 (13.2) & 2504 (12.9) \\
\hline Overweight & 9193 (22.4) & 4898 (22.7) & $4295(22.1)$ \\
\hline Obesity & 6474 (15.8) & $3450(16.0)$ & $3024(15.6)$ \\
\hline Morbid obesity & $583(1.4)$ & $291(1.3)$ & $292(1.5)$ \\
\hline Unknown & $18902(46.1)$ & $9849(45.6)$ & $9053(46.6)$ \\
\hline \multicolumn{4}{|c|}{ Socioeconomic deprivation index quintiles, $n(\%)$} \\
\hline Q1 & $7648(18.6)$ & $4188(19.4)$ & 3460 (17.8) \\
\hline Q2 & 7724 (18.8) & 3893 (18.0) & $3831(19.7)$ \\
\hline Q3 & 7511 (18.3) & $4128(19.1)$ & $3383(17.4)$ \\
\hline Q4 & 7706 (18.8) & 3942 (18.2) & $3764(19.4)$ \\
\hline Q5 & $7547(18.4)$ & 3832 (17.7) & $3715(19.1)$ \\
\hline Unknown & 2906 (7.1) & $1636(7.6)$ & $1270(6.5)$ \\
\hline \multicolumn{4}{|c|}{ Visits to PCC during the study period, $n(\%)$} \\
\hline 0 & 10163 (24.8) & 5183 (24.0) & $4980(25.6)$ \\
\hline 1 & $3295(8.0)$ & $1731(8.0)$ & $1564(8.0)$ \\
\hline 2 & 3246 (7.9) & $1748(8.1)$ & $1498(7.7)$ \\
\hline 3 & 3081 (7.5) & $1629(7.5)$ & $1452(7.5)$ \\
\hline 4 & 2841 (6.9) & 1509 (7.0) & $1332(6.9)$ \\
\hline$\geq 5$ & $18416(44.9)$ & $9819(45.4)$ & 8597 (44.3) \\
\hline \multicolumn{4}{|l|}{ Clinical risk group, $n(\%)$} \\
\hline Without dominant chronic illness & $15827(38.6)$ & 8459 (39.1) & 7368 (37.9) \\
\hline With dominant chronic illness & $23132(56.4)$ & $12136(56.1)$ & $10996(56.6)$ \\
\hline Unknown & $2083(5.1)$ & 1024 (4.7) & 1059 (5.5) \\
\hline
\end{tabular}

$P C C=$ primary care centre. $S D=$ standard deviation. percentage, and quantitative variables were shown as mean and standard deviation if normally distributed. The relationship between participation in the CCSPB and individuals' baseline characteristics, adjusted by study group, was assessed using a clustered standard errors method in a logistic regression model lone model for each characteristic).

The intention-to-treat analysis included all individuals randomised to each group, whereas the per-protocol analysis was limited to those attended by their GP or nurse within the follow-up period and without exclusion criteria for participating in the CCSPB. The study includes patient participants who were eligible for the population-based screening programme.

Nevertheless, some participants were excluded by the programme afterwards (Figure 1), since any of the exclusion criteria of the programme, unidentified before sending the invitation letter, was notified to the programme during the screening process lfor example, normal colonoscopy performed in last 5 years, colorectal cancer high risk by family history, presence of red flag symptoms).

In this study all initial patients included in the study in the intention-to-treat analysis were analysed; and excluded were those patients with any exclusion criteria identified by the programme during the screening process and also those patients who did not visit primary care during the followup period, in the per-protocol analysis (Figure 1).

Differences in participation between the intervention and control groups were established by a clustered standard errors method in a multiple logistic regression analysis: both unadjusted and adjusted by age, sex, smoking, alcohol consumption, body mass index, socioeconomic deprivation index, number of visits to primary care centre, and clinical risk group. Results of the analysis were reported as odds ratios (ORs) with $95 \%$ confidence intervals $(95 \% \mathrm{Cls})$. The adjusted analyses were performed including only those patients for whom all baseline variables were available (complete cases) and including all cases after missing data had been imputed according to the Imputation by the chained equation function of Stata. ${ }^{24}$ All results in Tables 2 and 3 have been analysed taking into account the correlation between outcomes of patients who belong to the same GP, using clustered robust standard errors method in a logistic regression analysis. More specifically, the Huber-White (also called Sandwich) standard errors method has been used. 
All analyses were performed using Stata (version 13.1).

\section{RESULTS}

After randomisation, $67 \mathrm{GPs}$ and their respective 21619 patients were allocated to the intervention group, while 63 GPs and their 19423 patients were allocated as controls. As shown in Table 1, both groups were similar with respect to baseline characteristics. Overall, 20.9\% of activated alerts were responded by primary care professionals. Adjusting for primary care frequentation (that is excluding the

\begin{tabular}{|c|c|c|c|c|c|}
\hline Characteristics & $\begin{array}{c}\text { Total } \\
n=41042\end{array}$ & $\begin{array}{c}\text { Non-participation } \\
n=23307\end{array}$ & $\begin{array}{c}\text { Participation } \\
n=17735\end{array}$ & OR $(95 \% \mathrm{CI})^{\mathrm{b}}$ & $P$-value ${ }^{\mathrm{b}}$ \\
\hline \multicolumn{6}{|l|}{ Study group, $n[\%]$} \\
\hline Control & $19423(47.3)$ & $11227(57.8)$ & 8196 (42.2) & $1^{c}$ & \\
\hline Intervention & $21619(52.7)$ & 12080 (55.9) & $9539(44.1)$ & 1.08 (0.97 to 1.20$)$ & 0.146 \\
\hline \multicolumn{6}{|l|}{ Sex, $n(\%)$} \\
\hline Male & $19039(46.4)$ & 11287 (59.3) & $7752(40.7)$ & $1^{c}$ & \\
\hline Female & $22003(53.6)$ & $12020(54.6)$ & $9983(45.4)$ & $1.21(1.16$ to 1.26$)$ & $<0.001$ \\
\hline \multicolumn{6}{|l|}{ Age groups, years, $n(\%)$} \\
\hline $50-54$ & $11991(29.2)$ & 7231 (60.3) & 4760 (39.7) & $1^{c}$ & \\
\hline $55-59$ & $10765(26.2)$ & $6155(57.2)$ & $4610(428)$ & $1.13(1.07$ to 1.20$)$ & $<0.001$ \\
\hline $60-64$ & $9910(24.2)$ & 5278 (53.3) & $4632(46.7)$ & $1.32(1.23$ to 1.41$)$ & $<0.001$ \\
\hline $65-69$ & $8376(20.4)$ & $4643(55.4)$ & $3733(44.6)$ & 1.21 (1.12 to 1.30$)$ & $<0.001$ \\
\hline \multicolumn{6}{|l|}{ Smoking, $n(\%)$} \\
\hline Never smokers & 14028 (48.3) & $7184(51.2)$ & 6844 (48.8) & $1^{c}$ & \\
\hline Smokers & 6908 (23.8) & $4268(61.8)$ & 2640 (38.2) & 0.65 (0.61 to 0.70 ) & $<0.001$ \\
\hline Ever smokers & 8105 (27.9) & $3946(48.7)$ & 4159 (51.3) & $1.10(1.01$ to 1.20$)$ & 0.020 \\
\hline \multicolumn{6}{|l|}{ Alcohol consumption, $n(\%)$} \\
\hline Non drinkers & 16352 (61.8) & 8675 (53.1) & $7677(46.9)$ & $1^{c}$ & \\
\hline Low-risk drinkers & 9349 (35.3) & $4723(505)$ & $4626(49.5)$ & $1.10(1.04$ to 1.17$)$ & 0.001 \\
\hline High-risk drinkers & $760(2.9)$ & $463(60.9)$ & $297(39.1)$ & $0.73(0.62$ to 0.86$)$ & $<0.001$ \\
\hline \multicolumn{6}{|l|}{ Body mass index, $n(\%)$} \\
\hline Low weight & $539(2.4)$ & $335(62.1)$ & 204 (37.9) & $1^{c}$ & \\
\hline Normal weight & $5351(24.2)$ & 2784 (52.0) & 2567 (48.0) & 1.51 (1.24 to 1.84$)$ & $<0.001$ \\
\hline Overweight & $9193(41.5)$ & 4641 (50.5) & $4552(49.5)$ & $1.61(1.34$ to 1.94$)$ & $<0.001$ \\
\hline Obesity & $6474(29.2)$ & $3382(50.2)$ & 3092 (47.8) & 1.50 (1.24 to 1.82$)$ & $<0.001$ \\
\hline Morbid obesity & $583(2.6)$ & 346 (59.3) & $237(40.7)$ & 1.12 (0.88 to 1.43 ) & 0.356 \\
\hline \multicolumn{6}{|c|}{ Socioeconomic deprivation index quintiles, $n(\%)$} \\
\hline Q1 & $7648(20.1)$ & $4659(60.9)$ & $2989(39.1)$ & $1^{c}$ & \\
\hline Q2 & $7724(20.2)$ & 4272 (55.3) & $3452(44.7)$ & 1.27 (1.16 to 1.39$)$ & $<0.001$ \\
\hline Q3 & $7511(19.7)$ & $3786(50.4)$ & $3725(49.6)$ & 1.53 (1.41 to 1.68$)$ & $<0.001$ \\
\hline Q4 & 7706 (20.2) & $4169(54.1)$ & $3537(45.9)$ & $1.33(1.21$ to 1.46$)$ & $<0.001$ \\
\hline Q5 & $7547(19.8)$ & $4761(63.1)$ & 2786 (36.9) & 0.92 (0.82 to 1.03 ) & 0.168 \\
\hline \multicolumn{6}{|c|}{ Visits to PCC during the study period, $n(\%)$} \\
\hline 0 & $10163(24.8)$ & 7222 (71.1) & 2941 (28.9) & $1^{c}$ & \\
\hline 1 & $3295(8.0)$ & $2023(61.4)$ & $1272(38.6)$ & 1.53 (1.39 to 1.69$)$ & $<0.001$ \\
\hline 2 & $3246(7.9)$ & $1881(57.9)$ & $1365(42.1)$ & 1.75 (1.59 to 1.92$)$ & $<0.001$ \\
\hline 3 & 3081 (7.5) & $1647(53.5)$ & 1434 (46.5) & 2.10 (1.92 to 2.30$)$ & $<0.001$ \\
\hline 4 & 2841 (6.9) & $1462(51.5)$ & 1379 (48.5) & 2.28 (2.04 to 2.54 ) & $<0.001$ \\
\hline$\geq 5$ & $18416(44.9)$ & 9072 (49.3) & 9344 (50.7) & 2.48 (2.31 to 2.66 ) & $<0.001$ \\
\hline \multicolumn{6}{|l|}{ Clinical risk group, $n(\%)$} \\
\hline Without dominant chronic illnes & s $15827(40.6)$ & $9090(57.4)$ & $6737(42.6)$ & $1^{c}$ & \\
\hline With dominant chronic illness & $23132(59.4)$ & 12465 (53.9) & $10667(46.1)$ & $1.15(1.10$ to 1.21$)$ & $<0.001$ \\
\hline
\end{tabular}

$P C C=$ primary care centre.${ }^{a}$ Based on intention-to-treat analysis. ${ }^{b}$ Each row is a logistic regression model adjusted by study group with clustered robust standard error method. 'Reference group. individuals that did not visit their primary care centre during the follow-up period) this figure rises to $27.5 \%$.

\section{Intention-to-treat analysis}

Participation in the CCSPB of individuals allocated to the intervention group was not significantly higher than that of those allocated to the control group $(44.1 \%$ versus $42.2 \%$ respectively) (OR $1.08,95 \% \mathrm{Cl}=0.97$ to $1.20, P=0.146$ ) (Table 2). No statistically significant improvements in colorectal cancer screening participation were found when clustering and other confounding variables were accounted for in the analysis (Tables 2 and 3).

\section{Per-protocol analysis}

When the analysis was restricted to those individuals who could benefit from the electronic reminder, the participation rate was higher, but not statistically significant, in the intervention group (OR $1.09,95 \% \mathrm{Cl}=0.99$ to 1.19; $P=0.065$ ) (Table 3). After adjusting for potential confounders, either in complete cases (OR 1.11, $95 \% \mathrm{Cl}=1.02$ to 1.22 , $P=0.018)$ or on missing-data imputation (OR $1.08,95 \% \mathrm{Cl}=1.01$ to $1.15 ; P=0.033$ ) (Table 3), colorectal cancer screening uptake showed a statistically significant increase in the intervention group.

\section{DISCUSSION}

\section{Summary}

These results show that an electronic alert in patients' primary care EMRs was not associated with a statistically significant increased participation in the first round of an organised, FIT-based colorectal cancer screening programme. Nevertheless, when the analysis was restricted to those individuals attending their primary care professional during the follow-up period and fulfilling the screening programme entry criteria, a beneficial effect lup to $11 \%$ increase in participation) was observed.

\section{Strengths and limitations}

To the authors' knowledge, this is the first study evaluating the impact of an electronic reminder in primary care to increase uptake in an organised, population-based colorectal cancer screening programme. Strengths of this study include a large sample size, its randomised design, and an accurate interpretation of results based on both intention-to-treat and per-protocol analyses by adjusting for cluster effect and potential confounding factors.

In addition, this trial included a broadly representative sample of the population at average risk of developing colorectal 


\begin{tabular}{|c|c|c|c|c|}
\hline & $n$ & OR & $95 \% \mathrm{Cl}$ & $P$-value \\
\hline \multicolumn{5}{|c|}{ Intention-to-treat analysis } \\
\hline \multicolumn{5}{|l|}{ Unadjusted } \\
\hline Control group & 19423 & $1^{\mathrm{a}}$ & & \\
\hline Intervention group & 21619 & 1.08 & 0.97 to 1.20 & 0.146 \\
\hline \multicolumn{5}{|c|}{ Adjusted (only complete cases) } \\
\hline Control group & 8185 & $1^{\mathrm{a}}$ & & \\
\hline Intervention group & 9394 & 1.09 & 0.99 to 1.19 & 0.064 \\
\hline \multicolumn{5}{|c|}{ Adjusted (missing-data imputationc) $^{\mathrm{c}}$} \\
\hline Control group & 19423 & $1^{\mathrm{a}}$ & & \\
\hline Intervention group & 21619 & 1.06 & 0.98 to 1.14 & 0.129 \\
\hline \multicolumn{5}{|l|}{ Per-protocol analysis ${ }^{d}$} \\
\hline \multicolumn{5}{|l|}{ Unadjusted } \\
\hline Control group & 13431 & $1^{\mathrm{a}}$ & & \\
\hline Intervention group & 15308 & 1.09 & 0.99 to 1.19 & 0.065 \\
\hline \multicolumn{5}{|c|}{ Adjusted (only complete cases) } \\
\hline Control group & 7100 & $1^{\mathrm{a}}$ & & \\
\hline Intervention group & 8181 & 1.11 & 1.02 to 1.22 & 0.018 \\
\hline \multicolumn{5}{|c|}{ Adjusted $^{b}$ (missing-data imputationc) } \\
\hline Control group & 13431 & $1^{\mathrm{a}}$ & & \\
\hline Intervention group & 15308 & 1.08 & 1.01 to 1.15 & 0.033 \\
\hline \multicolumn{5}{|c|}{$\begin{array}{l}\text { OR = odds ratio. Control group is the reference category. }{ }^{a} \text { Reference category. }{ }^{b} \text { Adjusted by sex, age, smoking, } \\
\text { alcohol consumption, body mass index, socioeconomic deprivation index, number of visits to primary care } \\
\text { centre, and clinical risk group. 'Missing-data imputation for smoking, alcohol consumption, body mass index, } \\
\text { socioeconomic deprivation index, and clinical risk group. }{ }^{d} \text { Performed in patients who attended a primary } \\
\text { care centre at least once during the study period and without CCSPB exclusion criteria identified during the } \\
\text { screening process. }\end{array}$} \\
\hline
\end{tabular}

cancer, in terms of age, sex, socioeconomic status, and comorbidity, as well as an evaluation of several colorectal cancer risk factors (that is, smoking, alcohol, and obesity), which allowed for the impact of the alert to be assessed in each subset of individuals. The study was also conducted under conditions of routine clinical practice and, therefore, its results may be applicable to countries with a public health system in which population-based colorectal cancer screening programmes involve primary care professionals.

However, the authors are aware of some limitations of the study. There were larger-than-expected pre-randomisation exclusions. Nevertheless, as most of them were due to administrative issues (patients with a primary care professional not assigned to a participating centre) or the primary care professional's reluctance to participate in the study, it is unlikely that they may represent any selection bias.

In addition, colorectal cancer screening participation differs, depending on the approach and test offered; ${ }^{25}$ results of this study are limited to organised, FIT-based programmes; these are the most widely implemented in Europe, ${ }^{26}$ but including only one test or approach could, nonetheless, be considered a limitation. Finally, because of socioeconomic differences identified between the participating centres la recognised determinant of screening participation) the GP was the unit of randomisation; as a consequence, a negligible interprofessional contamination cannot definitively be ruled out as intervention and control professionals coexisted in the same centres.

\section{Comparison with existing literature}

There is growing desire in Europe to improve participation in organised colorectal cancer screening programmes specifically, as most countries do not reach recommended European guidelines figures., ${ }^{27,28}$ To achieve this goal, several strategies and interventions have been proposed lfor example, postal or telephone reminders, including the GP's signature in the invitation letter, or mailing FIT samples), but they are influenced by the characteristics of such programmes. ${ }^{29}$ Interventions based on invitation letter or GP involvement, among others, were consistently effective in screening participation when organised programmes were compared with opportunistic approaches. ${ }^{30}$

Prompting health professionals during a medical encounter aims to reduce missed opportunities to recommend, order, or 
deliver cancer screening services.31,32 A systematic review, including 61 clinical trials in which various alerts were evaluated, found an average increase of $12-14 \%$ in delivering preventive care measures. ${ }^{20}$ However, results of a recent Cochrane review specifically assessing the usefulness of synchronous electronic reminders suggest that their impact is small or modest (median absolute improvement of $4.2 \%$ ), and probably less than would have been expected in the context of an increasingly wider use of EMRs. ${ }^{33}$ In eight comparisons, individuals allocated to the intervention group experienced a median absolute improvement of $2.5 \%$, in comparison with the $1.9 \%$ found in the current study. However, this review included only one study in the context of colorectal cancer screening. ${ }^{34}$

Another systematic review on the effectiveness of provider reminders to increase breast, cervical, and colorectal cancer screening performance concluded that they have a beneficial effect with respect to the use of mammography, cytology, flexible sigmoidoscopy, and faecal occult blood testing. ${ }^{31}$ In the last scenario, six studies were evaluated, with an average increase of $10.5 \%$ on screening. ${ }^{31}$ Finally, a recent meta-analysis, including five clinical trials assessing the role of physician reminders to increase participation in colorectal cancer screening using faecal occult blood testing, found a nonstatistically significant higher participation in the intervention group. ${ }^{18}$ In one of the evaluated studies, an electronic reminder was specifically assessed, although this was done in a non-population-based scenario in which both faecal occult blood testing and endoscopic tests were offered. ${ }^{35}$ These authors found that screening among individuals whose physicians received an electronic alert did not significantly increase compared with those whose doctors did not receive the reminder $141.9 \%$ versus $40.2 \%$ respectively). ${ }^{35}$ These results were very similar to those found in this current study, despite the fact that the GPs' response rate to the alert (20.9\%) was lower than in others. ${ }^{36}$

\section{Implications for research and practice}

The use of an electronic alert in individuals primary care EMRs was not associated with a statistically significant increased uptake of an organised, FIT-based colorectal cancer screening programme that had a moderate baseline participation rate. Nevertheless, when the analysis was restricted to those individuals who could benefit from the electronic reminder, colorectal cancer screening uptake showed a statistically significant increase in the intervention group, after adjusting for potential confounders. Strategies to improve primary care professionals' responses to the alert could improve the potential benefits of electronic reminders (for example, by involving primary care professionals in the alert design and working, limit alerts to a shorter period of time, arranging specific primary care visits addressing colorectal cancer screening, thus avoiding competition with other medical consultations, or improve communication and information feedback between primary care and populationbased screening programmes).

Given that low-intensity interventions targeting a large number of individuals may have a relevant impact on health promotion according to the five-dimension RE-AIM framework (reach, efficacy/ effectiveness, adoption, implementation, and maintenance), ${ }^{37}$ further research on this topic should still be considered.

The authors have declared no competing interests.

\section{Acknowledgements}

The authors thank all members of Barcelona Colorectal Cancer Screening Programme's executive committee for the design and implementation of the study, the Catalan Health Institute's Primary Care Services Information System for its participation in the design and management of the electronic medical record alert, and all health professionals and patients in the participating centres. A list of the members of the PROCOLON group are available from the authors.

\section{Discuss this article}

Contribute and read comments about this article: bjgp.org/letters 


\section{REFERENCES}

1. Ferlay J, Soerjomataram I, Ervik M, et al. GLOBOCAN 2012 v1.0, Cancer Incidence and Mortality Worldwide: IARC CancerBase No.11. Lyon, France International Agency for Research on Cancer. http://globocan.iarc.fr/Pages/ fact_sheets_cancer.aspx [accessed 25 May 2016].

2. Levin B, Lieberman DA, McFarland $B$, et al. Screening and surveillance for the early detection of colorectal cancer and adenomatous polyps, 2008 a joint guideline from the American Cancer Society, the US Multi-Society Task Force on Colorectal Cancer, and the American College of Radiology. Gastroenterology 2008; 134(5): 1570-1595.

3. Mandel JS, Church TR, Bond JH, et al. The effect of fecal occult-blood screening on the incidence of colorectal cancer. N Engl J Med 2000; 343(22): 1603-1607.

4. Hewitson P, Glasziou P, Watson E, et al. Cochrane systematic review of colorectal cancer screening using the fecal occult blood test (hemoccult): an update. Am J Gastroenterol 2008; 103(6): 1541-1549.

5. Atkin WS, Edwards R, Kralj-Hans I, et al. Once-only flexible sigmoidoscopy screening in prevention of colorectal cancer: a multicentre randomised controlled trial. Lancet 2010; 375(9726): 1624-1633.

6. Heitman SJ, Hilsden RJ, Au F, et al. Colorectal cancer screening for averagerisk North Americans: an economic evaluation. PLoS Med 2010; 7(11): e1000370.

7. Segnan N, Patrick J, von Karsa L (eds). European guidelines for quality assurance in colorectal cancer screening and diagnosis. 1st edn. Luxembourg: Publications Office of the European Communities, 2010.

8. Australian Institute of Health and Welfare and the Australian Government Department of Health. Analysis of colorectal cancer outcomes for the Australian National Bowel Cancer Screening Program. Asia Pac J Clin Oncol 2016; 12: 22-32.

9. American Cancer Society. Colorectal Cancer Facts \& Figures 2014-2016. 2014. $\mathrm{http} / / / \mathrm{w} w \mathrm{w}$.cancer.org/acs/groups/content/documents/document/acspc-042280. pdf laccessed on 23 May 2016).

10. Peris M, Espinàs JA, Muñoz L, et al. Lessons learnt from a population-based pilot programmeme for colorectal cancer screening in Catalonia (Spain). J Med Screen 2007: 14(2): 81-86.

11. Pérez Riquelme F, Navarro Sánchez C, Chirlaque López MD, et al. [Report on the prevention of colon and rectal cancerin the Murcia region]. In: Pérez Riquelme F, Cruzado Quevedo J, Gutiérrez García JJ, eds. [The prevention of colon and rectal cancer in the Murcia region]. [In Spanish] Murcia: Consejería de Sanidad de la Región de Murcia, 2008. Serie informes No: 50.

12. Málaga López A, Salas Trejo D, Sala Felis T, et al. [Programme of screening for colorectal cancer in the Valencia community, Spain. results of the first round (2005-2008)]. [In Spanish]. Rev Esp Salud Pública 2010; 84(6): 731-743.

13. Brugos-Llamazares V, González de Aledo Linos A, Vada-Sánchez J, et al. [Results of the screening program for colorectal cancer screening in Cantabria, Spain, during the period November 2008-March 2010]. [In Spanish]. Rev Esp Salud Pública 2010; 84(6): 757-770.

14. Portillo I, Idígoras I, Ojembarrena $\mathrm{E}$, et al. [Main results of the colorectal cancer screening program in the Basque Country (Spain)]. [In Spanish]. Gac Sanit 2013; 27(4): 358-361.

15. Burón A, Grau J, Andreu M, et al. [Colorectal Cancer Early Screening Program of Barcelona, Spain: indicators of the first round of a program with participation of community pharmacies]. [In Spanish]. Med Clin (Barc) 2015; 145(4): 141-146.

16. Cardarelli R, Thomas JE. Having a personal health care provider and receipt of colorectal cancer testing. Ann Fam Med 2009; 7(1): 5-10.

17. Ramos M, Taltavull M, Piñeiro $P$, et al. [Attitudes of primary health care users to a colorectal cancer screening program]. [In Spanish]. Gac Sanit 2013; 27(6): 516-520.

18. Siddiqui MRS, Sajid MS, Khatri K, et al. The role of physician reminders in faecal occult blood testing for colorectal cancer screening. Eur J Gen Pract 2011; 17(4): 221-228.

19. Pignone MP, Lewis CL. Using quality improvement techniques to increase colon cancer screening. Am J Med 2009: 122(5): 419-420.

20. Dexheimer JW, Talbot TR, Sanders DL, et al. Prompting clinicians about preventive care measures: a systematic review of randomized controlled trials. $J$ Am Med Inform Assoc 2008; 15(3): 311-320

21. Guiriguet-Capdevila C, Muñoz-Ortiz L, Rivero-Franco I, et al. Can an alert in primary care electronic medical records increase participation in a population-based screening programme for colorectal cancer? COLO-ALERT, a randomised clinical trial. BMC Cancer 2014: 14: 232

22. Domínguez-Berjón F, Borrell C, Cano-Serral G, et al. [Constructing a deprivation index based on census data in large Spanish cities (the MEDEA Project)]. [In Spanish]. Gac Sanit 2008; 22(3): 179-187.

23. Averill RF, Goldfield NI Eisenhandler J, et al. Development and evaluation of clinical risk groups. Final Report to the National Institutes of Standards and Technology, US Department of Commerce. http://solutions.3m.

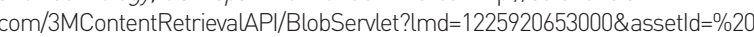
$1180606514454 \&$ assetType=MMM_Image\&blobAttribute=ImageFile laccessed 23 May 2016)

24. Royston P. Multiple imputation of missing values. Stata J 2004; 4(3): 227-241.

25. Quintero E, Castells A, Bujanda L, et al. Colonoscopy versus fecal immunochemical testing in colorectal-cancer screening. N Engl J Med 2012; 366(8): 697-706

26. Schreuders EH, Ruco A, Rabeneck L, et al. Colorectal cancer screening: a global overview of existing programmes. Gut 2015; 64(10): 1637-1649.

27. Senore C, Inadomi J, Segnan N, et al. Optimising colorectal cancer screening acceptance: a review. Gut 2015; 64(7): 1158-1177.

28. Von Karsa L, Patnick J, Segnan N. European guidelines for quality assurance in colorectal cancer screening and diagnosis. First edition - executive summary. Endoscopy 2012; 44(Suppl 3): SE1-8.

29. Camilloni L, Ferroni E, Cendales BJ, et al. Methods to increase participation in organised screening programs: a systematic review. BMC Public Health 2013; 13: 464.

30. Ferroni $\mathrm{E}$, Camilloni $\mathrm{L}$, Jimenez B, et al. How to increase uptake in oncologic screening: a systematic review of studies comparing population-based screening programmes and spontaneous access. Prev Med 2012; 55(6): 587-596.

31. Baron RC, Melillo S, Rimer BK, et al. Intervention to increase recommendation and delivery of screening for breast, cervical, and colorectal cancers by healthcare providers: a systematic review of provider reminders. Am J Prev Med 2010; 38(1): 110-117.

32. Sabatino SA, Lawrence B, Elder R, et al. Effectiveness of interventions to increase screening for breast, cervical, and colorectal cancers: nine updated systematic reviews for the guide to community preventive services. Am J Prev Med 2012; 43(1): 97-118

33. Shojania KG, Jennings A, Mayhew A, et al. The effects of on-screen, point of care computer reminders on processes and outcomes of care. Cochrane Database Syst Rev 2009: 8(3): CD001096.

34. Tape TG, Campbell JR. Computerized medical records and preventive health care: success depends on many factors. Am J Med 1993; 94(6): 619-625.

35. Sequist TD, Zaslavsky AM, Marshall R, et al. Patient and physician reminders to promote colorectal cancer screening: a randomized controlled trial. Arch Intern Med 2009; 169(4): 364-371.

36. Nease DE Jr, Ruffin MT 4th, Klinkman MS, et al. Impact of a generalizable reminder system on colorectal cancer screening in diverse primary care practices: a report from the prompting and reminding at encounters for prevention project. Med Care 2008; 46 (9 Suppl 1): S68-73.

37. Glasgow RE, Vogt TM, Boles SM. Evaluating the public health impact of health promotion interventions: the RE-AIM framework. Am J Public Health 1999. 89(9): 1322-1327. 